35th Symposium on The Search for Extraterrestrial Intelligence (SETI) - The Next Steps (A4.)

SETI I - Technical Aspects (1.)

Author: Mr. Andrew Howard

Harvard University, Cambridge, MA, United States, howard@frank.harvard.edu

Mr. Paul Horowitz

Harvard University, Cambridge, MA 02138, United States, horowitz@physics.harvard.edu Mr. Curtis Mead

Harvard University, Cambridge, MA, United States, cmead@fas.harvard.edu Mr. Pratheev Sreetharan

Harvard University, Cambridge, MA, United States, sreethar@fas.harvard.edu Mr. Jason Gallicchio

Harvard University, Cambridge, MA, United States, jason@physics.harvard.edu Mr. Steve Howard

Harvard University, Cambridge, MA, United States, srhoward@fas.harvard.edu Dr. Charles Coldwell

Harvard University, Cambridge, MA, United States, coldwell@frank.harvard.edu Mr. Joseph Zajac

Harvard-Smithsonian, Cambridge, MA, United States, zac@cfa.harvard.edu Mr. Alan Sliski

Harvard University, Cambridge, MA, United States, asliski@comcast.net

\title{
INITIAL RESULTS FROM HARVARD ALL-SKY OPTICAL SETI
}

\begin{abstract}
We report initial results from the Harvard/Planetary Society all-sky search for pulsed optical signals from other civilizations, which saw "first light" on 11 April, 2006 after six years of planning and construction. To survey of the Northern sky $(-20 \mathrm{deg} ;$ declination $i$ +60 deg), our 1.8-meter spherical f/2.5 optical telescope images a $1.6 \mathrm{deg}$ x $0.2 \mathrm{deg}$ patch of sky on two matched focal planes with a total of 1024 photomultiplier tube pixels. Each pair of pixels images the same 2.3 square arcminute patch of sky, and fast electronics filters the incoming visual band light for nanosecond pulses. Coincident optical flashes in a pair of pixels triggers one of 32 PulseNet full-custom chips to record the pulse profiles at nanosecond resolution. This experiment is an all-sky, kilopixel evolution of our targeted search. Its meridian transit survey mode requires 150 clear nights to cover the Northern sky with 1 minute dwell time per source point. Focus in this talk will be given to describing the capabilities of the all-sky search instrument, the first few months' observations, and constraints that these observations place on the density of pulsed optical signals in the galaxy.
\end{abstract}

